\title{
ESTUDIO COMPARATIVO DEL CONOCIMIENTO DE LAS TÉCNICAS DE REPRODUCCIÓN ASISTIDA EN ESTUDIANTES DE MEDICINA DE UNIVERSIDADES CON DIFERENTES IDEARIOS ÉTICOS Y HUMANÍSTICOS COMPARATIVE STUDY OF THE KNOWLEDGE OF IVF IN MEDICAL STUDENTS OF UNIVERSITIES WITH DIFFERENT ETHICAL AND HUMANISTIC VALUES ESTUDO COMPARATIVO DO CONHECIMENTO DAS TÉCNICAS DE REPRODUÇÃO ASSISTIDA EM ESTUDANTES DE MEDICINA DE UNIVERSIDADES COM DIFERENTES IDEÁRIOS ÉTICOS E HUMANÍSTICOS
}

Guillermo Cantú-Quintanilla ${ }^{1}$

Carlos Vidal-Sentíes ${ }^{2}$ Francisco Javier Marcó-Bach ${ }^{3}$ Fernando Camargo-Prieto ${ }^{4}$ Nuria Aguiñaga-Chiñas ${ }^{5}$

Daniela Contreras-Estrada ${ }^{6}$

DOI: 10.5294/pebi.2020.24.2.4

\section{PARA CITAR ESTE ARTÍCULO / TO REFERENCE THIS ARTICLE / PARA CITAR ESTE ARTIGO}

Cantú-Quintanilla G, Vidal-Sentíes C, Marcó-Bach FJ, Camargo-Prieto F, Aguiñaga-Chiñas N, Contreras-Estrada D. Estudio comparativo del conocimiento de las técnicas de reproducción asistida en estudiantes de medicina de universidades con diferentes idearios éticos y humanísticos. Pers Bioet. 2020;24(2):166-176. DOI: https://doi.org/10.5294/pebi.2020.24.2.4

1 凶https://orcid.org/0000-0002-3493-2207. Universidad Panamericana, Centro Interdisciplinario de Bioética, México. gcantu@up.edu.mx

2 https://orcid.org/0000-0002-4290-4265. Universidad Panamericana, Escuela de Psicología, México.cvidal@up.edu.mx

3 https://orcid.org/0000-0003-3069-2354. Universidad Panamericana, Escuela de Medicina, México.jmarco@up.edu.mx

4 https://orcid.org/0000-0001-5848-4640. Universidad Panamericana, Escuela de Medicina, México.

5 https://orcid.org/0000-0002-3339-5809. Universidad Panamericana, Escuela de Psicología, México.naguinaga@up.edu.mx

6 https://orcid.org/0000-0001-7907-5870. Universidad Panamericana, Escuela de Medicina, México.dcontreras@up.edu.mx

RECEPCIÓN: 04/09/2019

ENVÍO A PARES: 17/09/2019

APROBACIÓN POR PARES: 02/03/2020

ACEPTACIÓN: 05/08/2020 


\section{RESUMEN}

La fertilización in vitro (FIV) se sigue presentando como una solución relativamente sencilla para producir un embarazo sin dar a conocer la problemática médica y ética que esta representa. Este proyecto consistió en realizar una encuesta a los estudiantes de tres facultades de Medicina de la Ciudad de México, con diferentes idearios éticos y humanísticos, con el objetivo de evaluar el conocimiento sobre las técnicas de fecundación in vitro y sus implicaciones éticas. Se aplicó una encuesta a 404 alumnos de medicina. Los alumnos de escuelas con ética personalista tuvieron un mayor reconocimiento de los principios bioéticos implicados en el uso de estas técnicas. El conocimiento de la técnica y de las cuestiones éticas implicadas en la reproducción asistida podría mejorar la sensibilidad de los alumnos de medicina ante dilemas éticos que se presenten en su ejercicio profesional.

Palabras clave (Fuente: DeCS): Fertilización in vitro; bioética; estudiantes de medicina; personal de salud; ética

\section{Abstract}

In vitro fertilization (IVF) continues to be presented as a relatively simple solution to produce a pregnancy without exposing the medical and ethical problems that this represents. This project consisted of conducting a survey to medical students from three medical schools in Mexico City with different ethical and humanistic ideas with the aim of evaluating knowledge about IVF techniques and their ethical implications. The survey was applied to 404 medical students. Students in schools with personalistic ethics had a greater recognition of the bioethical principles involved in the use of these techniques. Knowledge of the technique and ethical issues involved in assisted reproduction could improve the sensitivity of medical students to ethical dilemmas that arise in their professional practice.

KeY wORDs (Source: DeCS): Fertilization in vitro; bioethics; students, medical; health personnel; ethics.

\section{Resumo}

A fertilização in vitro (FIV) continua sendo apresentada como uma solução relativamente simples para produzir uma gestação sem dar a conhecer a problemática médica e ética que ela representa. Esse projeto consistiu em realizar uma pesquisa com estudantes de três faculdades de Medicina da Cidade do México, com diferentes ideários éticos e humanísticos, com o objetivo de avaliar o conhecimento sobre as técnicas de fecundação in vitro e suas repercussões éticas. Foi aplicada uma pesquisa a 404 alunos de Medicina. Os alunos de escolas com ética personalista tiveram um maior reconhecimento dos princípios bioéticos implicados no uso dessas técnicas. O conhecimento da técnica e das questões éticas implicadas na reprodução assistida poderia melhorar a sensibilidade dos alunos de Medicina ante dilemas éticos que são apresentados em seu exercício profissional.

Palavras-chave (Fonte: DeCS): Fertilização In Vitro; bioética; estudantes de medicina; pessoal de saúde; ética. 


\section{INTRODUCCIÓN}

En 1976 y 1978 se informó el primer embarazo después de la fertilización in vitro (FIV) de un óvulo humano y el primer nacimiento de un bebé de FIV, respectivamente. Desde entonces, la FIV y sus modificaciones han logrado aproximadamente siete millones de embarazos en todo el mundo. Estos procedimientos se conocen genéricamente como técnicas de reproducción asistida (TRA) e incluyen la inyección intracitoplasmática de espermatozoides (ICSI), la transferencia intrafalopiana de gametos (GIFT) y la transferencia intrafalopiana de cigoto (ZIFT). En Estados Unidos, cerca del 1,5\% de todos los nacimientos y el $20 \%$ de todos los nacimientos múltiples son el resultado de la FIV; la tasa es mayor cuando se consideran todos los tipos de concepción médicamente asistida.

Con el tiempo, la tasa de éxito y de indicaciones de las TRA ha aumentado. Sin embargo, las preocupaciones sobre el resultado de estos embarazos acompañan su creciente prevalencia. La investigación en este campo es complicada por la necesidad de distinguir entre los efectos de TRA en los resultados de la descendencia y otros factores de confusión o mediación. Además, estos resultados están influenciados por los cambios tecnológicos en el desempeño de TRA, así como por los cambios en la atención obstétrica y neonatal con el tiempo (1).

La FIV es un procedimiento diseñado para producir un embarazo como resultado directo de una intervención. En general, los ovarios son estimulados por una combinación de medicamentos para la fertilidad y, luego, uno o más ovocitos se aspiran de los folículos ováricos (2). Estos se fertilizan en el laboratorio (in vitro), después de lo cual, uno o más embriones se transfieren a la cavidad uterina (3).
LA FIV ES UN PROCEDIMIENTO DISEÑADO PARA PRODUCIR UN EMBARAZO COMO RESULTADO DIRECTO DE UNA INTERVENCIÓN. EN GENERAL, LOS OVARIOS SON ESTIMULADOS POR UNA COMBINACIÓN DE MEDICAMENTOS PARA LA FERTILIDAD Y, LUEGO, UNO O MÁS OVOCITOS SE ASPIRAN DE LOS FOLÍCULOS OVÁRICOS.

Han surgido nuevas investigaciones que consideran aspectos éticos relevantes (4), que abordan el problema de la infertilidad desde un punto de vista más integral (5). Sin embargo, la FIV se sigue presentando como una solución relativamente sencilla para la infertilidad, sin dar a conocer toda la problemática (3) asociada a ella. La mayoría de los estudios formales hablan sobre las malformaciones de los niños nacidos con FIV (6-8), a pesar del control de calidad preimplantatorio, del diagnóstico prenatal y de un registro de malformaciones clínicas en los sitios donde se practican, que no parece coincidir con la realidad (9-11). Otros estudios se centran en los problemas de salud de la madre, como el síndrome de hiperestimulación ovárica (12), o los riesgos obstétricos que califican a los embarazos por FIV siempre como de alto riesgo (13). El niño concebido también está expuesto a mayores riesgos obstétricos que los que proceden de un embarazo normal, al menos con problemas un $25 \%$ más frecuentes (14). Existen algunos estudios que compilan información de otros muchos sobre diversas complicaciones $(15,16)$.

En cuanto a su eficacia, según los últimos datos publicados por el Comité Internacional de Monitoreo de las 
Técnicas de Reproducción Asistida, la probabilidad a nivel mundial de tener un hijo se calcula en un $20 \%$ (17), la mayor parte de las veces con al menos tres intentos de transferencia de embriones, aunque la tasa varía según los países. Los embriones restantes, que no se implantan, se usan para investigación u otros fines lucrativos (18). En 2012, el New England Journal of Medicine publicó una revisión hecha en Adelaida, Australia, en 308.974 nacimientos, de los cuales 6.163 (2\%) fueron por reproducción asistida. Se encontró que hay una tasa de riesgo mucho más alta de defectos de nacimiento en los nacidos por ICSI (FIV más inyección intracitoplasmática de espermatozoides) que en los provenientes de un embarazo natural (19).

Además, son cada vez más numerosos los estudios que muestran la escasa eficacia, las complicaciones de salud -tanto de la madre como del hijo- y el alto costo de las técnicas de FIV (20). Sin embargo, la población en general tiene la idea de que es la mejor o una muy buena opción para las parejas infértiles e ignoran los problemas de salud y éticos que estos métodos conllevan (21).

Desde el punto de vista ético, contribuye a causar más confusión la bondad, al menos aparente, del deseo de procrear, lo que, sumado al desconocimiento de los muchos embriones humanos que hay que sacrificar para que nazca uno, y de las complicaciones de salud de la madre y del hijo, llevan a formar una opinión positiva sobre estas técnicas que infortunadamente, como sucede con otros muchos temas, se apoya en la falta de conocimiento.

La ignorancia sobre las complicaciones de salud y éticas de las técnicas de FIV, aun por personas con interés en adquirir una formación profesional en ciencias de la salud, es actual y universal.
Al ver esta problemática desde un punto de vista ético, la ética personalista se desarrolla a partir de una perspectiva filosófica con un énfasis basado en el reconocimiento de la dignidad de la persona, a diferencia de la bioética laica o principialista, basada en una ética liberal consencuencialista y emotivista que se queda en la consideración de los principios tradicionales de beneficencia, no maleficencia, justicia y autonomía (22).

Por tanto, el objetivo de la encuesta a estudiantes de medicina de todos los niveles de licenciatura fue averiguar si hay un desconocimiento entre personas con distintos grados de formación en ciencias de la salud y, al mismo tiempo, contribuir a disminuirlo al propiciar una reflexión sobre el tema en los estudiantes de medicina de las universidades mencionadas. Se espera provocar en algunos de ellos la inquietud de conocer más sobre el tema y que estudien a fondo las técnicas de FIV, su eficacia y sus complicaciones.

A la fecha, no hemos encontrado ningún otro trabajo -encuesta, entrevista, etc.- que aborde el tema con los estudiantes de medicina de la ciudad. Por lo anterior, el interés de esta investigación fue determinar el grado de conocimiento de las técnicas de FIV, de su eficacia y sus complicaciones éticas y de salud por los estudiantes de medicina en las universidades encuestadas. Por tanto, nos preguntamos, ¿hay un distinto nivel de conocimiento médico y bioético sobre la fecundación in vitro con transferencia de embrión (Fivet) en universidades laicas o en universidades humanistas?

\section{BIOÉTICA PERSONALISTA Y BIOÉTICA PRINCIPIALISTA}

La finalidad de la bioética personalista es promover el bien íntegro de la persona humana, vértice de lo creado, 
eje y centro de la vida social; esta corriente de pensamiento no es igual al individualismo subjetivista, aquel que considera la capacidad de decisión como parte de la naturaleza del hombre.

Los principios de la bioética personalista son:

1. Defensa de la vida física: la vida física es considerada el valor fundamental de la persona; sin un cuerpo físico la persona no existe y, en consecuencia, no tiene libertad. La vida antecede a la libertad. Cuando la libertad que suprime la vida es una libertad que se suprime a sí misma.

2. Totalidad: la corporalidad de la persona constituye una totalidad. A partir de esto se justifica el principio terapéutico donde se permite tratar una parte del cuerpo cuando no se puede curar la totalidad.

3. Libertad y responsabilidad: la persona es libre siempre que busque un bien para sí misma y los demás; el mundo es una responsabilidad humana. No existe la libertad sin responsabilidad.

4. Sociabilidad y subsidiariedad: la organización está para beneficiar a las personas, pero estas también deben retribuirle a ella. Además, implica ayudar a quienes no pueden ayudarse por sí mismos, la sociedad es una verdadera sociedad cuando es solidaria.

El principialismo, por su parte, se puede entender a partir de sus cuatro principios fundamentales:

1. Autonomía: en la clínica implica que el paciente será quien tenga prioridad en la toma de decisiones, procurando siempre salvaguardar su dignidad. Con este principio se deja en el pasado la postura paternalista médica.
2. Beneficencia: el beneficio de los demás siempre deberá ser el fin; en la clínica se buscará siempre hacer todo por el bien del paciente.

3. No maleficencia: conocido como primum non nocere, nos obliga a no dañar al otro.

4. Justicia: la sociedad es una parte fundamental en el acto médico; por ello, todas las personas merecen el mismo respeto y derecho a la vida, la salud y la equidad de los recursos sanitarios.

García (23) propone cinco justificaciones válidas en caso de la eventual desobediencia de algún principio.

1. Tener razones más valiosas para obedecer una norma emergente.

2. El objetivo moral debe ser realista.

3. No se puede reemplazar por una alternativa moral.

4. La infracción debe ser la que menores consecuencias provoque.

5. Se deben minimizar las consecuencias a toda costa.

Existe mucha ambigüedad en la metodología que este autor plantea; deja de lado la jerarquía de los principios y, además, los contextualiza, lo que supone un conflicto. Valdría la pena, entonces, cuestionar qué implica esta postura ante los conflictos éticos del final de la vida, por ejemplo; se abre así el espacio para el relativismo.

El Iluminismo buscó resarcir este conflicto al mitificar el uso de la razón universal; propone que se deje de considerar una sola razón y validar múltiples posturas y corrientes filosóficas que replantean a la ética. La bioética resulta del Iluminismo y es considerada como 
"el desarrollo de una estructura laica de racionalidad en una era de incertezas”. Rechaza entonces las posturas clásicas y teológicas.

De acuerdo con García (23), según lo planteado por Engelhardt, las virtudes morales ideales en una sociedad son la tolerancia, la liberalidad y la prudencia, a diferencia de la moral occidental que pretende justificar, a través de la razón, cualquier moralidad; esto parecería algo insostenible.

Así, podemos considerar la autonomía como el lenguaje universal de cualquier expresión moral. Sin embargo, no se debe tener una conducta pasiva o de obediencia ante los principios éticos (24).

\section{MÉTODO}

Buscamos evaluar los conocimientos médicos y bioéticos sobre los métodos de FIV de los estudiantes de medicina de una universidad donde se aplica una bioética laica y dos universidades donde se aplica una bioética personalista, de todos los niveles de la licenciatura en Medicina; asimismo, mediante la encuesta, se intenta estimular la inquietud por conocer mejor la situación actual de las técnicas de FIV.

Esta es una investigación original con diseño transversal, analítico y descriptivo. El tamaño de muestra fue basado en los alumnos inscritos en la carrera de medicina, en cualquier nivel de la licenciatura, en una universidad donde se aplica una bioética laica y dos universidades donde se aplica una bioética personalista. Las encuestas fueron contestadas por escrito en dos universidades y en una de enfoque personalista se hicieron en una plataforma digital.

\section{INSTRUMENTO}

Se elaboró un cuestionario con 20 preguntas de selección múltiple, que admiten una sola respuesta válida de tres opciones, con base en el grado de conocimiento y la opinión ética de los encuestados sobre la FIV. A cada pregunta se le dio un puntaje por pregunta correcta, se podía obtener un máximo de 20 puntos y un mínimo de cero. En el caso de la universidad personalista, el cuestionario se envió en línea a todos alumnos inscritos en la carrera de Medicina, en cualquier nivel de la licenciatura; a las otras dos universidades los cuestionarios se enviaron en físico.

Evitamos las preguntas que suponen un conocimiento muy especializado y se formularon de tal manera que el sentido común ayudase a descubrir la opción correcta. Para probar si hubo diferencias estadísticamente significativas entre los grupos de comparación, se realizó la prueba chi-cuadrado con corrección de Yates, y la prueba exacta de Fisher (2 colas), a través del programa estadístico SPSS/PC v22.0 (Statistical Package for Social Sciences, Chicago, IL) y JMP v14.

\section{RESULTADOS}

Participaron 404 alumnos de tres escuelas de medicina de la ciudad, dos con bioética personalista y una con bioética laica, 151, 103 y 150 respectivamente; 137 hombres y 267 mujeres, con una edad promedio de 20,8 años. De los estudiantes, 158 son de primer año, 87 de segundo, 75 de tercero, 47 de cuarto, 3 de quinto y 34 de sexto año. Han cursado la materia de Ginecología 272 alumnos y 132 no lo han hecho. Las diferencias en los puntajes fueron significativas por año de estudios, edad y haber cursado la materia de Ginecología (tabla 1). 
Tabla 1. Características sociodemográficas de la muestra estudiada

\begin{tabular}{|c|c|c|c|c|c|c|}
\hline & & $\begin{array}{l}\text { Población total } \\
\qquad \mathrm{n}=\mathbf{4 0 4}\end{array}$ & $\begin{array}{c}\text { Universidad } \\
\text { personalista } 1 \\
n=151\end{array}$ & $\begin{array}{c}\text { Universidad } \\
\text { laica } \\
\mathbf{n}=\mathbf{1 5 0}\end{array}$ & $\begin{array}{c}\text { Universidad } \\
\text { personalista } 2 \\
\text { n }=103\end{array}$ & $\mathbf{p}$ \\
\hline \multicolumn{2}{|c|}{$\begin{array}{l}\text { Edad promedio } \\
\text { Años (rango) }\end{array}$} & $\begin{array}{c}20,8 \\
(18-33)\end{array}$ & $\begin{array}{c}20 \\
(18-33)\end{array}$ & $\begin{array}{c}21 \\
(18-29)\end{array}$ & $\begin{array}{c}20 \\
(17-25)\end{array}$ & 0,0096 \\
\hline $\begin{array}{l}\text { Sexo } \\
\mathrm{N}(\%)\end{array}$ & $\begin{array}{c}\text { Hombres } \\
\text { Mujeres }\end{array}$ & $\begin{array}{l}137(34) \\
267(66)\end{array}$ & $\begin{array}{c}46(30) \\
105(70)\end{array}$ & $\begin{array}{c}46(31) \\
104(69)\end{array}$ & $\begin{array}{l}45(44) \\
58(56)\end{array}$ & 0,5774 \\
\hline Año de medicina & $\begin{array}{l}1^{\circ} \\
2^{\circ} \\
3^{\circ} \\
4^{\circ} \\
5^{\circ} \\
6^{\circ}\end{array}$ & $\begin{array}{c}158(39) \\
87(22) \\
75(18) \\
47(12) \\
3(1) \\
34(8)\end{array}$ & $\begin{array}{c}34(23) \\
54(36) \\
36(24) \\
19(12) \\
3(2) \\
5(3)\end{array}$ & $\begin{array}{c}91(60) \\
0(0) \\
22(15) \\
19(13) \\
0(0) \\
18(12)\end{array}$ & $\begin{array}{c}33(32) \\
33(32) \\
17(16) \\
9(9) \\
0(0) \\
11(11)\end{array}$ & $<0,0001$ \\
\hline $\begin{array}{c}\text { Materia de } \\
\text { Ginecología cursada }\end{array}$ & $\begin{array}{l}\text { Sí } \\
\text { No }\end{array}$ & $\begin{array}{l}132(33) \\
272(67)\end{array}$ & $\begin{array}{l}21(14) \\
130(86)\end{array}$ & $\begin{array}{l}99(66) \\
51(34)\end{array}$ & $\begin{array}{l}12(12) \\
91(88)\end{array}$ & 0,0223 \\
\hline \multicolumn{2}{|c|}{$\begin{array}{l}\text { Puntaje de la encuesta sobre } 100 \\
\text { Promedio (rango) }\end{array}$} & $\begin{array}{c}56,3 \\
(10-100)\end{array}$ & $\begin{array}{c}58,9 \\
(10-100)\end{array}$ & $\begin{array}{c}45 \\
(20-80)\end{array}$ & $\begin{array}{c}65 \\
(15-100)\end{array}$ & $<0,0001$ \\
\hline
\end{tabular}

Fuente: elaboración propia.

Las preguntas donde hubo más aciertos de las tres escuelas fueron la 1, 2, 4, 6, 7, 8, 9, 11, 15, 1618 y 20 (con un puntaje de $345,314,345,246,211,358,322,259,228$, 235,197 y 265 respectivamente). Estas preguntas se refieren a qué son las técnicas de FIV; los embriones que se transfieren y los que se congelan; los embriones que presentan alguna alteración o enfermedad son eliminados o utilizados con fines de investigación; la técnica tiende a producir más malformaciones en los niños que en el embarazo natural; mayor probabilidad que tiene un embrión obtenido por FIV de morir durante la gestación; dar opciones a los que se implantan y las dificultades para conseguir la implantación; las complicaciones obstétricas más frecuentes de la FIV: embarazo ectópico, embarazo múltiple y aborto espontáneo; las complicaciones de los tratamientos hormonales hiperestimulantes para los ova- rios; las implicaciones psicológicas para el padre, la madre y el niño por la sobreprotección y la falta de identidad si este proviene de donadores de gametos; este embarazo siempre se considera de alto riesgo, donde aumenta la prevalencia de problemas genéticos y congénitos en el niño concebido; el costo del mismo, entre 50 y 160 mil pesos MN (entre 2.200 y 7.200 dólares).

Las preguntas donde hubo menos aciertos de las tres escuelas fueron la $3,5,10,12,13,14,17$ y 19 (117, 160, 151, 122, 182, 134, 173 y 105 respectivamente). Estas preguntas se refieren al porcentaje de éxito, que es menor del $25 \%$; el $90 \%$ de los embriones mueren; a nivel internacional, la media de probabilidad de tener un hijo por FIV es menor del 30\%; la presencia de cariotipos con anomalías y malformaciones congénitas; el deseo de ser 
padres sobre el bien de los hijos; la pertinencia del uso de embriones humanos producidos por FIV en investigación; la FIV nunca será tan exigente como la natural, en la cual solo uno de 200 millones de espermatozoides fecunda el óvulo; y la información resulta escasa, con tendencia a minimizar los problemas que puedan presentarse.

Respecto al reconocimiento de los principios de la bioética en relación con la FIV, se observa que las escuelas con bioética personalista tienen un mayor reconocimiento, comparado con la escuela de bioética laica. De forma particular, la dignidad es el principio que se reconoce con más frecuencia por los alumnos de las tres escuelas; en contraste, la justicia es el que menos se reconoce en toda la muestra (figura 1).

\section{Figura 1. Reconocimiento de los principios de la bioética por escuela}

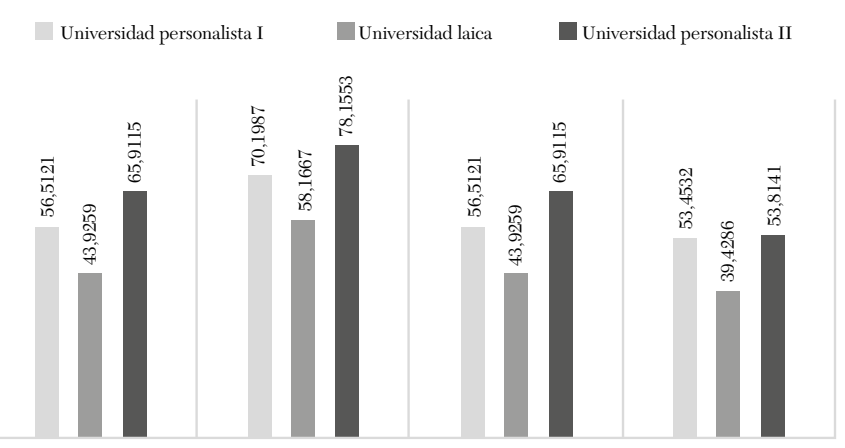

Fuente: elaboración propia.

$\mathrm{Al}$ contrastar el reconocimiento de los principios de la bioética con el grado que cursan los participantes, el principio con más reconocimiento es la autonomía, en cinco de los seis grados académicos $\left(\mathrm{p}=0,0437^{*}\right)$. Se observó que, en primer año existe un menor reconocimiento de los demás principios, y que el reconocimiento de estos tiene un aumento gradual con el paso de los grados escolares. Respecto a la no maleficencia, se encontró que existe un aumento significativo en su reconocimiento conforme los alumnos cursan grados académicos superiores $\left(\mathrm{p}<0,0001^{*}\right)$; similar al fenómeno que sucede con la justicia $\left(\mathrm{p}<0,0001^{*}\right)$ y la dignidad $\left(\mathrm{p}=0,0016^{*}\right)$ (figura 2).

\section{Figura 2. Reconocimiento de los principios de la bioética por grado de estudio}

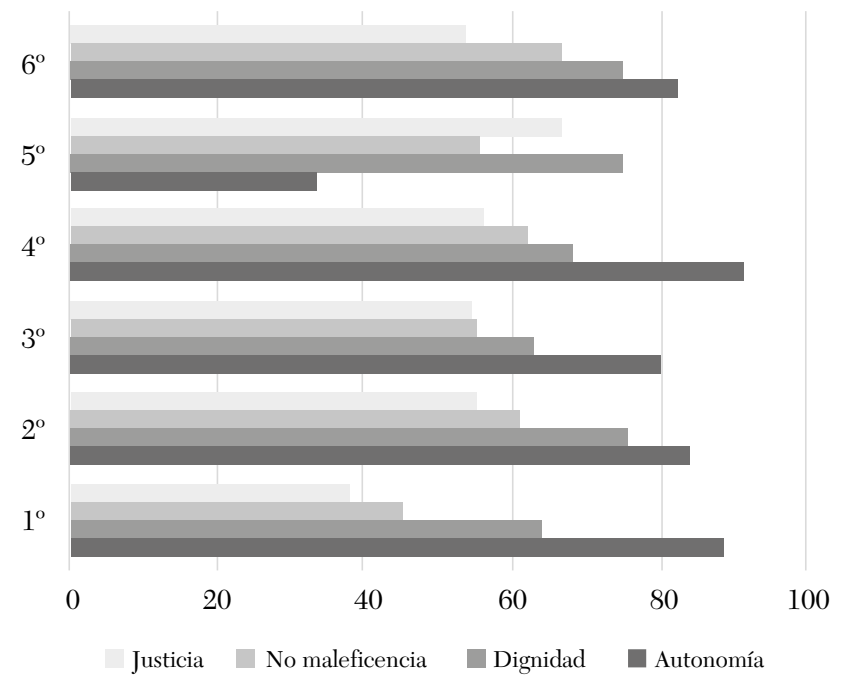

Fuente: elaboración propia.

\section{DISCUSIÓN}

El trabajo realizado nos confirma que aún existe un amplio desconocimiento de los aspectos éticos de las técnicas de reproducción asistida por parte de los estudiantes de Medicina evaluados.

Es necesario recordar que el cigoto originado se debe respetar en su integridad biológica y nunca debe ser instrumentalizado o usado como medio, ya que toda persona humana es un fin en sí misma. No todo lo técnicamente posible es éticamente admisible. 
Por otro lado, se encontró también que el reconocimiento de las cuestiones éticas relacionadas con la FIV es mayor por parte de los alumnos de las universidades que se acogen a una ética personalista al compararse con aquellos de la universidad que adopta una ética laica, lo que supone cierta influencia del tipo de ética en la formación de los alumnos. Es importante destacar que también se observan diferencias en el conocimiento del tema según si los alumnos han cursado o no la materia de Ginecología y Obstetricia, lo que permite suponer que una vez estos tienen más información respecto a las técnicas, consecuencias y prevalencia, también tienen una consideración más amplia de los principios de la bioética y las cuestiones éticas que se ven involucradas en el proceso de la reproducción asistida.

Por otro lado, se olvida con frecuencia que en las técnicas de reproducción asistida el hijo formado tiene el derecho inalienable de conocer a sus padres biológicos. La selección de los embriones más “aptos" termina en la crioconservación, primero, y luego en la eliminación voluntaria de los sobrantes o su donación para fines de investigación. De la implantación de varios embriones para conseguir mayor eficacia de las técnicas de reproducción asistida se sigue la reducción embrionaria o eliminación de los no deseados.

El anonimato puede causar importantes problemas psicológicos en la propia pareja y en el hijo cuya identidad queda oscurecida para siempre.

Un nuevo filtro es el diagnóstico prenatal para el aborto de fetos con malformaciones ocasionadas durante el proceso de la fertilización. La naprotecnología se ve como una alternativa más viable para atender los procesos de infertilidad, sin las consecuencias negativas que tienen las técnicas de reproducción asistida, la FIV y la transferencia de embriones.

\section{CONCLUSIÓN}

Una bioética personalista tiene como eje rector la dignidad de la persona, su naturaleza y su trascendencia.

En una bioética principialista, las directrices propuestas pueden entrar en conflicto y no hay una jerarquía entre ellas, con lo que su aplicación tiene una cierta indeterminación y ambigüedad que podrían justificar posturas incluso contradictorias.

Los alumnos de Medicina que reciben un entrenamiento personalista están en condiciones de poder apreciar la vida del no nacido y su carácter inviolable, mientras que, en un enfoque de los principios, con mucha frecuencia laicista -que desconoce la dimensión natural de la espiritualidad humana- con facilidad se menosprecia y se da un valor relativo a la vida en gestación, como se pudo comprobar en el estudio realizado.

\section{LIMITACIONES}

Todo instrumento tiene limitaciones en cuanto al número de preguntas o la extensión del cuestionario. En una próxima investigación se podría preguntar a los estudiantes si conocen alguna alternativa a la Fivet. Además, se les podría cuestionar sobre su conocimiento de la reducción embrionaria y sus implicaciones bioéticas. ${ }^{7}$

7 La reducción embrionaria ( $\mathrm{RE}$ ) es la interrupción selectiva del desarrollo de uno o varios fetos en el primer trimestre de un embarazo múltiple de alto riesgo (trillizos, cuatrillizos o más). Es una práctica que se ha desarrollado con el auge de las técnicas de reproducción asistida (25). 


\section{REFERENCIAS}

1. Paulson MD. Pregnancy outcome after assisted reproductive technology. Waltham, MA: UpToDate Inc. Disponible en: https:/www.uptodate.com/contents/pregnancy-outcomeafter-assisted-reproductive-technology

2. Paulson, MD. In vitro fertilization. UpToDate. Waltham, MA: UpToDate Inc. Disponible en: https://www.uptodate.com/ contents/in-vitro-fertilization

3. Ombelet $\mathrm{W}$ et al. Infertility and the provision of infertility medical services in developing countries. Hum Reprod Update. 2008;14(6):605-21. DOI: http://doi.org/10.1093/humupd/ dmn042

4. Brecina PR, Zhao Y. The ethical, legal and social issues impacted by modern assisted reproductive technologies. Obstet Gynecol Int. 2012:686253. DOI: https://doi. org/10.1155/2012/686253

5. Marcó J. Dilemas pediátricos de la reproducción asistida. En Véliz Pintos RA, editor. Bioética y humanismo para pediatría. México: Graphimedic; 2018. p. 515-25.

6. Mitsiakos K et al. A case of Adams-Oliver Syndrome following in vitro fertilization. Human Reprod. 2009;24:1529-30. DOI: https://doi.org/10.1093/humrep/dep096

7. Hansen M et al. Assisted reproductive technology and birth defects: A systematic review and meta-analysis. Hum Reprod Update. 2013;19(4):330-53. http://doi.org/10.1093/humupd/ dmt006

8. Lu Y, Wang $\mathrm{N}$ and Jin F. Long-term follow-up of children conceived through assisted reproductive technology. J Zhejiang Univ Sci B. 2013;14(5):359-371. DOI : http://doi. org/10.1631/jzus.B1200348

9. Qin J et al. Pregnancy-related complications and adverse pregnancy outcomes in multiple pregnancies resulting from assisted reproductive technology: A meta-analysis of cohort studies. Fertil Steril. 2015;103(6):1492-508. DOI: https://doi. org/10.1016/j.fertnstert.2015.03.018

10. Fortunato A, Tosti E. The impact of in vitro fertilization on health of the children: An update. Eur J Obstet Gynecol. Reprod Biol. 2011;154:125-9. DOI: https://doi.org/10.1016/j.ejogrb.2010.10.012

11. Odom LN, Segars J.Imprinting disordersandassisted reproductive technology. Curr Opin Endocrinol Diabetes Obes. 2010;17:51722. https://doi.org/10.1097/MED.0b013e32834040a3

12. Goisis A et al. Medically assisted reproduction and birth outcomes: A within-family analysis using finnish population registers. Lancet. 2019;393(10177):1225-32. DOI: https://doi. org/10.1016/S0140-6736(18)31863-4

13. Nastri CO et al. Ovarian hyperstimulation syndrome: Pathophysiology, staging, prediction and prevention. Ultrasound Obstet Gynecol. 2015;45(4):377-93. DOI: https://doi. org/10.1002/uog.14684

14. Pandey $\mathrm{S}$ et al. Obstetric and perinatal outcomes in singleton pregnancies resulting from IVF/ICSI: A systematic review and meta-analysis. Hum Reprod Update. 2012;18(5):485-503. DOI: https://doi.org/10.1093/humupd/dms018

15. Chen M, Heilbronn LK. The health outcomes of human offspring conceived by assisted reproductive technologies (ART). J Dev Orig Health Dis. 2017;8(4):388-402. DOI: http://doi. org/10.1017/S2040174417000228

16. Kawwass JF, Badell ML. Maternal and fetal risk associated with assisted reproductive technology. Obstet Gynecol. 2018;132(3):763-72. DOI: http://doi.org/10.1097/ AOG.0000000000002786

17. Qin J et al. Assisted reproductive technology and the risk of pregnancy-related complications and adverse pregnancy outcomes in singleton pregnancies: A meta-analysis of cohort studies. Fertil Steril. 2016;105(1):73-85. DOI: https://doi. org/10.1016/j.fertnstert.2015.09.007 
18. Dyer S et al. International Committee for Monitoring Assisted Reproductive Technologies world report: Assisted Reproductive Technology 2008, 2009 and 2010. Hum Reprod. 2016; 31(7):1588-609. DOI: https://doi.org/10.1093/humrep/dew082

19. Spar DL. The baby business: How money, science and politics drive the commerce of conception. Boston: Harvard Business School; 2006. DOI: http://doi.org/10.1172/JCI29554

20. Davies MJ et al. Reproductive technologies and the risk of birth defects. N Engl J Med. 2012;366(19):1803-13. DOI: http:// doi.org/10.1056/NEJMc1206859

21. López Moratalla N, Huera Zepeda A, Bueno López D. Riesgos para la salud de los nacidos por las técnicas de fecundación asistida. La punta de un iceberg. Rev Cuad Bioét. 2012;XXIII(78). Disponible en: http://aebioetica.org/revistas/2012/23/78/467.pdf
22. Pérez-Tamayo, R. La ley, la ética médica y los trasplantes. Rev Invest Clin. 2005;57(2):170-6. Disponible en: https://www. medigraphic.com/pdfs/revinvcli/nn-2005/nn052j.pdf

23. García JJ. Bioética personalista y bioética principialista. Perspectivas. Cuad Bioét. 2013;24(1):67-76. Disponible en: http:// aebioetica.org/revistas/2013/24/80/67.pdf

24. García-Sánchez E. ¿Es un acto de amor humano la fecundación in vitro? Una propuesta de análisis ético. Cuad Bioét. 2014;XXV(83):169-83. Disponible en: https://www.bioeticaweb.com/wp-content/uploads/2014/07/amoryfivet.pdf

25. Sánchez Ramírez N, Carrillo Bermúdez L, Sanabria Arias AM, \& Olivella Mejía L. Reducción embrionaria en embarazo gemelar. Rev Cubana Obstet Gineco. 2019; 45(2). Disponible en: http://scielo.sld.cu/scielo.php?script=sci_arttext\&pid=S0138600X2019000200015 